\title{
MOOC - MASSIVE OPEN ONLINE COURSE: UMA PERSPECTIVA PARA O ACESSO DEMOCRÁTICO AO CONHECIMENTO
}

\author{
SÃO PAULO/SP MAIO/2018
Leonardo Manoel Mendes - Faccamp - leonardomanoelmendes@gmail.com
Elaine Barbosa de Figueiredo - Faccamp - ebfigueiredo@anhembi.br
Eliana Pereira da Silva - Faccamp - eliana.pereiras@gmail.com
Renan Aleixo Paganatto - Faccamp - renan.ccn@gmail.com
Júlio Alberto Vansan Gonçalves - Faccamp - julio@vansan.com.br \\ Cristina Becker Matos Nabarro - Faccamp - cristina.nabarro@cps.sp.gov.br
}

Tipo: Investigação Científica (IC)

Natureza: Relatório Final de Pesquisa

Categoria: Métodos e Tecnologias

Setor Educacional: EDUCAÇÃO MÉDIA E TECNOLÓGICA, EDUCAÇÃO SUPERIOR, EDUCAÇÃO CORPORATIVA, EDUCAÇÃO CONTINUADA EM GERAL

\section{RESUMO}

Este trabalho tem por objetivo uma abordagem na utilização de MOOCs - Massive Open Online Course, como uma metodologia para o acesso democrático à educação. Os MOOCs por sua vez têm proporcionado nos últimos anos acesso ao conhecimento em diferentes áreas do conhecimento por meio das tecnologias de informação e comunicação TICs. O formato de formação em massa por meio dos MOOCs tem permitido capacitar pessoas em diferentes partes do mundo de forma gratuita, bastando apenas um dispositivo com acesso à internet para que possa usufruir de todo 0 conhecimento que deseja obter por meio das plataformas de educação a distância com MOOCs.

Palavras-chave: Palavras chaves: MOOC, Educação a distância - EAD, democratização do conhecimento

\section{AGRADECIMENTOS}

AGRADECEMOS A TODOS OS ENVOLVIDOS. 


\section{Introdução}

Os MOOCs têm proporcionado formação em massa e democrática a milhares de pessoas espalhados ao redor do mundo. O acesso aos cursos nesta modalidade de ensino é através de ambientes virtuais de aprendizagem ou plataformas de educação a distância com cursos de universidades renomadas dos EUA, Canadá, universidades europeias, asiáticas, assim como cursos ofertados por algumas universidades brasileiras como a Universidade de São Paulo - USP e a Universidade de Brasília - UNB. Os MOOCs ofertados pelas universidades, tem a finalidade de oferecer cursos de excelente qualidade em diferentes áreas do conhecimento. Os cursos disponíveis nas plataformas são gratuitos, porém em alguns casos para que o aluno possa receber o certificado é necessário pagar uma taxa. No entanto, a opção pelo certificado é uma escolha do aluno, uma vez que todo o conteúdo disponível para o curso é de acesso livre para que o aluno possa obter conhecimento sem que haja a necessidade de pagar pelo curso. Um MOOC é um curso on-line com a opção de inscrição gratuita e aberta, um currículo compartilhado publicamente e resultados abertos (MCAULEY ET AL. 2010).

Os MOOCs, surgiram em 2008 e até os dias atuais tem ganhado espaço e aceitação pela comunidade acadêmica e área corporativa. O sucesso dos MOOCs em grande parte é devido a sua flexibilidade em poder acessar os conteúdos disponíveis para cada curso ofertado por meio de diferentes dispositivos com acesso à internet, ou seja, computadores desktops, Notebooks, Tablets, smartphones, dentre outros dispositivos que permitam o acesso à internet para auxiliar o aluno durante a sua necessidade para obtenção do conhecimento. Este formato de curso permite ao aluno realizar cursos em diferentes partes do mundo por meio das plataformas de ensino de forma gratuita e sem a necessidade de deslocamento físico para uma sala de aula, tendo em vista que, todo acesso ao conhecimento é realizado através da internet para acessar as plataformas que de forma democrática oferecem formação e conhecimento para quem buscar ser aprimorar profissionalmente a cada dia.

De acordo com (Souza, Costa De, Márcio Vieira de; Simon 2014), o ser humano se caracteriza por ser, essencialmente, social e simbólico. Baseado no contexto dos autores é essencial que as pessoas busquem se socializar e se relacionar por meio de diferentes canais de comunicação e interação humana para que possa buscar conhecimento. Um destes canais que o ser humano na era da tecnologia pode utilizar ao seu favor são as plataformas de ensino em educação aberta e cursos no formato de MOOC. 
MOOC sigla em inglês - Massive Open Online Course que traduzindo para o português (Curso Online Aberto e Massivo). Os MOOCs surgiram em 2008 e desde o seu surgimento vem ganhando destaque em todo o mundo. Destaque este devido a sua aceitação em diferentes áreas de conhecimentos e principalmente nos cursos de formação que fazem uso da modalidade de educação à distância EaD. Para (Matta, Cláudia Eliane da; Figueiredo 2013), Mooc é um curso online que pode fazer uso de diferentes tipos de plataformas, aberto e gratuito e os cursos não exigem pré-requisitos para que os usuários possam se matricular nos cursos. Para (Silva, Junior, and Oliveira 2014), por se tratar de um elemento novo no universo da EaD, os MOOC ainda carecem de ajustem no modo como estão estruturados, isto é, em seus modelos de negócios. MOOC pode ser definido como um modelo que integra três elementos: a conectividade das redes sociais, o conhecimento de um especialista em determinada área e a coleção de recursos online abertos (MATTA, CLÁUDIA ELIANE DA; FIGUEIREDO 2013).

Para (MENDES, DA SILVA, and ORTOLANI 2017), Os MOOCs em si têm por objetivo promover a aprendizagem colaborativa em um ambiente virtual de aprendizagem por meio da internet e, proporcionar formação continuada em diferentes áreas de conhecimento e, na área da saúde. Baseado na abordagem do dos autores, os MOOCs podem ser utilizados para proporcionar acesso democrático a informação em diferentes áreas do conhecimento. Como citado a área da saúde, os MOOCs têm auxiliado para que os profissionais tenham acesso a informação de maneira flexível devido as suas rotinas de trabalho serem na maioria das vezes incompatíveis com horários de centros de formação tradicional.

Os MOOCs também podem considerado como uma educação aberta e flexível e de acordo com (Pereira 2015), a educação aberta pode se referir à educação flexível. Baseado neste contexto, o autor faz uma abordagem levando em consideração as necessidades de cada indivíduo que busca conhecimento por meio da educação aberta e livres das barreiras físicas, econômicas e sociais para que possam obter conhecimento de forma democrática.

\section{Educação a distância - EAD e os MOOCs}

A educação a distância nos últimos anos teve um elevado crescimento, isso devido aos recursos de tecnologias da informação e comunicação TICs por meio da internet. $O$ avanço tecnológico permite que indivíduos não fiquem reféns das barreiras físicas impostas pela educação tradicional, ou seja, a educação presencial dentro de uma sala de aula e sob a orientação de um professor. No modelo EAD, o cenário é diferente, ou seja, o aluno passa a ter flexibilidade de horários para ler os conteúdos disponíveis para 
cada disciplina ou módulo de um determinado curso. Esse modelo de educação exige que o aluno possua um autogerenciamento para que possa cumprir com os objetivos do curso em si.

De acordo com (Mattar 2011), o modelo de Educação a Distância (EaD) mais adotado no Brasil começa com um conteudista, que produz conteúdo que é trabalhado por um designer instrucional e um webdesigner, posteriormente disponibilizado para o aluno, que conta com o atendimento de um tutor. No modelo de educação a distância, os educadores devem buscar compreender os reais interesses dos seus alunos reais e, com base nessas informações, integrar atividades de aprendizagem que tenham relevância real para cada aluno. (MATTAR 2011). Nos últimos anos com o avanço da tecnologia da informação e a disponibilização de tais recurso permitiram o surgimento de uma gama de cursos EAD no formato de MOOC.

De acordo com (Litto 2014), aqueles que estudam a tecnologia educacional procuram a inovação apropriada (praticidade, eficiência, custo-benefício) de avanços tecnológicos a fim de não engessar regimentar, automatizar, ou desumanizar a aprendizagem. O autor também afirma que o propósito de tudo isso é para que haja uma maior compreensão, ou seja, o autor quis dizer que é fornecer para o aluno o alcance de diferentes fontes de informação independente das barreiras que poderão existir.

\section{Acesso democrático aos Moocs por meio de diferentes plataformas}

Desde o seu surgimento em 2008, os MOOCs vêm ganhando conhecimento e ampliando os horizontes no acesso democrático a educação em massa em diferentes áreas do conhecimento. Pelo mundo existem diversas plataformas que fornecem formação em massa e de excelente qualidade através de universidades renomadas e seus respectivos docentes. Para (Carla Beatris Valentini and Soares 2010), um MOOC é um curso online que utilizar diferentes plataformas, sendo aberto e gratuito, no entanto não é necessário que exista pré-requisitos para matrícula nos cursos.

Dentre as plataformas existentes, algumas ganham destaques quando se trata da formação em massa, sendo elas: Coursera, edX, Veduca, Khan Academy. Estas são as plataformas de ensino que oferecem cursos EAD em formato Mooc mais conhecidas do público brasileiro. Dentre as plataformas citadas, a Coursera é a maior plataforma de elearning relacionada aos MOOCs, na plataforma há 62 universidades e cerca de 2 mil cursos. Nesta plataforma, toda a formação é gratuita, no entanto, caso o aluno queria receber o certificado, este deverá pagar uma taxa. 


\section{Plataforma Veduca}

Veduca que uma plataforma que disponibiliza curso desenvolvidos pela Universidade de São Paulo - USP, Universidade de Brasília - UNB, é uma plataforma brasileira e dispõe de cursos nas áreas de física básica, probabilidade e estatística e bioenergética.

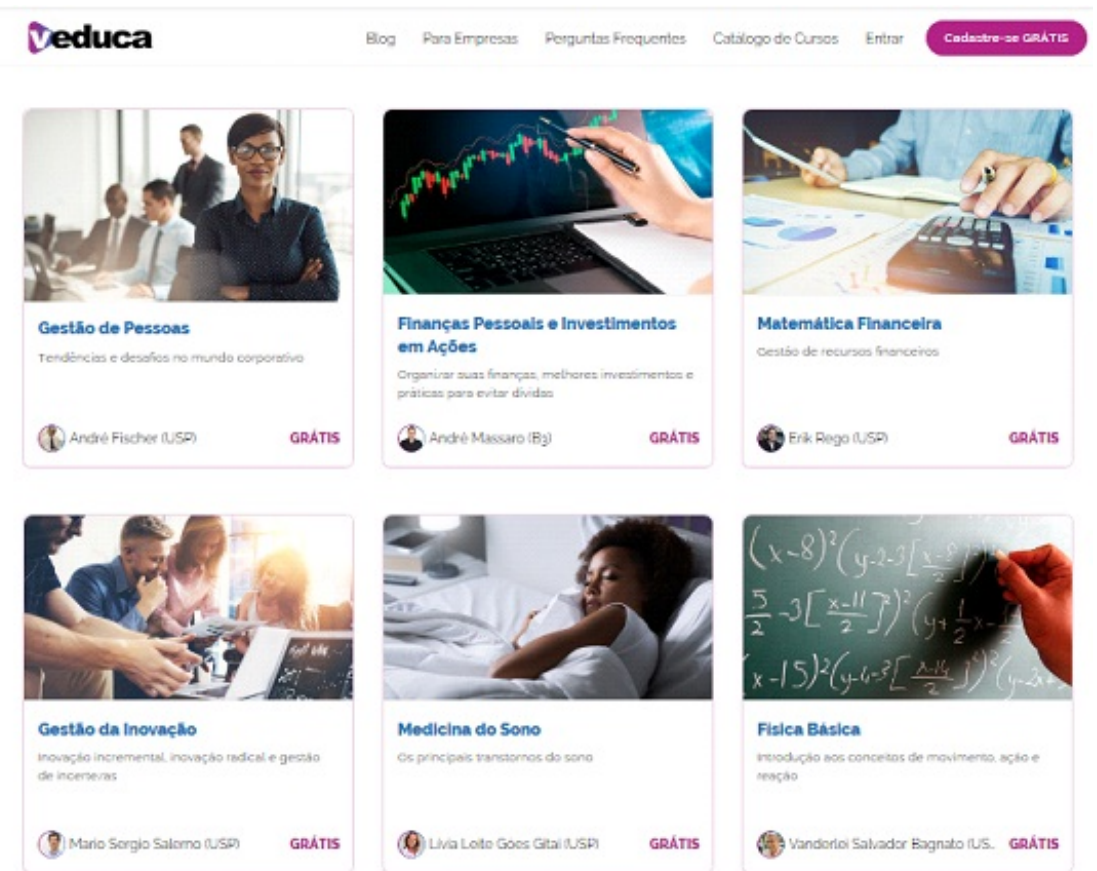

figura 1. Home Page - Veduca - https://veduca.org/

Na plataforma Veduca o aluno poderá escolher um ou mais cursos para que possa cursar. Para a realização da matrícula em qualquer um dos cursos disponíveis na plataforma, o aluno deverá efetuar um cadastro simples para que possa se autenticar e realizar a matrícula no curso desejado.

\section{Plataforma Coursera}

A Coursera é uma plataforma de ensino a distância com cursos de várias universidades renomadas em diferentes partes do mundo. Dentre os países com corsos são destacados os seguintes: Argentina, Australia, Belgium, Brazil, Canada, Chile, China, Colombia, Denmark, France, Germany, Hong, Kong, India, Israel, Italy, Japan, Mexico, Netherlands, Russia, Singapore, South Africa, South Korea, Spain, Sweden, Switzerland, Turkey, United Kingdom, United States.

De acordo com (Pappano 2012), universidades de elite estão fazendo uma parceria com a Coursera em um ritmo furioso. Dentre as universidades que oferecem cursos abertos 
em diferentes áreas do conhecimento destacam-se as universidades: ILLIONOIS, PENN, John Hopikins University, University of Michigan, Stanford University, San Diego, University of Copenhagen, Universidade de São Paulo - USP, Instituto Tecnológico de Aeronáutica - ITA, Universidade Estadual de Campinas - UNICAMP, Rutgers the State University of New Jersey, University of Copenhagen, dentre outras universidades que podem ser encontradas na plataforma.
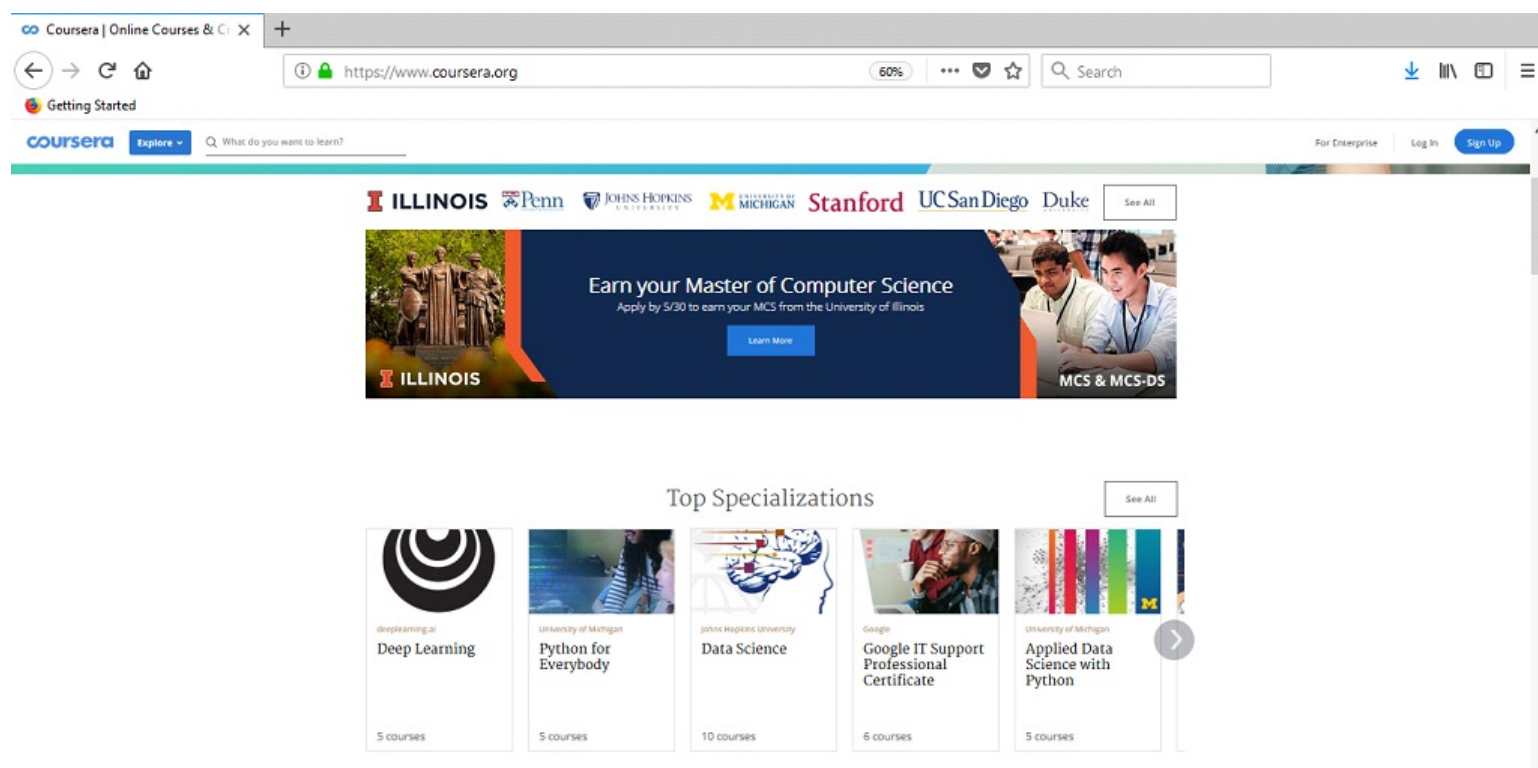

Figura 2. Home Page Cousera - https://www.coursera.org/

Os cursos disponíveis nas plataformas Coursera, edX e Khan Academy são curso disponíveis em grande maioria na língua inglesa, em alguns cursos existe a legenda para a língua portuguesa. A Khan Academy foi fundada em 2007 por Salman Khan, na plataforma podem ser encontrados curso em diferentes áreas como: Artes e humanidades, Computação, Economia e finanças, Matemática, Ciências e engenharia. Principais instituições parceiras são: NASA, a Academia de Ciências da Califórnia e o MIT. Um ponto muito importante que todos devem saber é que os alunos matriculados nos MOOCs fazem parte do grupo de estudantes matriculados na instituição (AIRES 2016).

\section{Plataforma edX}

A plataforma edX é uma plataforma de cursos desenvolvidos por universidades renomadas em todo o mundo. Os cursos são gratuitos e para acesso ao conteúdo os usuários devem efetuar um cadastro simples na plataforma para que possam se matricular nos respectivos cursos. Importante ressaltar que os cursos não exigem prérequisitos para que possam se matricular. 


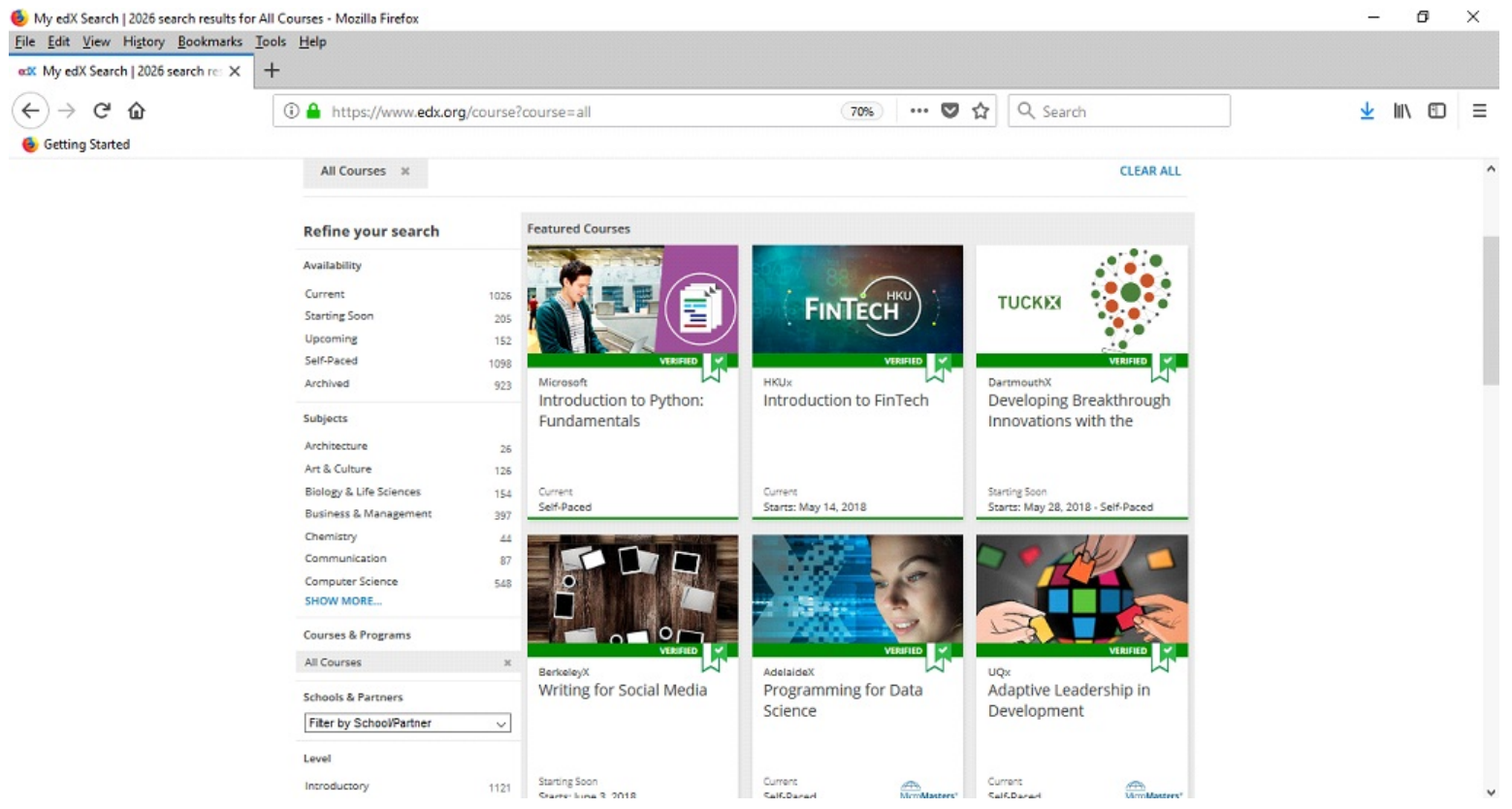

Figura 3. Home Page edX - https://www.edx.org/course?course=all

Além das plataformas citadas, os cursos no formato de Mooc são oferecidos por universidades em diversas partes do mundo. As universidades europeias, tais como: Université Nantes (França), Universidad Carlos III de Madrid (Espanha), Graz University of Technology e Universidade Aberta (Portugal), oferecem curso de formação em massa para todos que desejam obter conhecimentos e formação de qualidade sem que seja necessário pagar para ter acesso ao curso.

\section{Modelos de MOOCs oferecidos pelas plataformas}

Os MOOCs em geral são ofertados em diferentes plataformas de educação a distância, e cada uma das plataformas oferecem tanto curso no formato de vídeos, textos em slides, animações em HTML com recursos de áudio, dentre outros formatos que podem ser adotados para o acesso democrático ao conhecimento. Os MOOCs têm o objetivo de tornar o conhecimento mais acessível e de forma mais rápida e práticas paras os indivíduos que buscam este tipo de curso para o seu aprimoramento profissional ou pessoal.

De acordo com (Gonçalves 2013), o vídeo é cada vez mais um recurso educativo que pode ser adotado. No entanto, existem milhares de vídeos com diferentes temáticas disponíveis na internet que contribuem para o acesso democrático ao conhecimento.

\section{Conclusão}


O acesso democrático ao conhecimento por meio dos MOOCs tem se tornado mais evidente a cada dia, isso devido ao crescente número de universidades que vem aderindo a essa modalidade de compartilhamento de conhecimento por meio das plataformas digitais de ensino a distância. O avanço tecnológico tem permitido esse crescimento de curso e disseminação do conhecimento ao alcance de todos para que possam ser acessados através de dispositivos com acesso a internet.

Como visto, para acessar os cursos não é necessário pagar, ou seja, os cursos são disponibilizados de forma gratuita e não existe pré-requisitos para que as pessoas em diferentes partes do mundo possam acessar. Cabe ressaltar que, as pessoas que buscam este tipo de curso não têm vínculo de aluno com a universidade que está ofertando o curso. outro ponto importante é que após o termino do curso o aluno caso opte por receber o certificado de conclusão, este terá que pagar uma taxa para obter o mesmo.

Em geral os MOOCs são alternativas fáceis e rápidas de obtenção de conhecimento de qualidade oferecidos de forma gratuita por universidades renomadas em diferentes partes do mudo, sem as limitações das barreiras físicas, econômicas e sociais presentes na vida de grande parte da população mundial.

\section{Referências}

AIRES, Luísa. 2016. "E-Learning, Educação Online e Educação Aberta: Contributos Para Uma Reflexão Teórica e-Learning, Online Education and Open Education: A Contribution to a Theoretical Approach." RIED V 19 (1): 253-69.

CARLA Beatris Valentini, Eliana Maria do Sacramento, and Soares. 2010. "Aprendizagem Em Ambientes Virtuais" 331. goo.gl/6igG9V

GONÇALVES, Bruno Miguel Ferreira. 2013. "MOOC e B-Learning : Uma Proposta Para o Mestrado Em TIC Na Educação e Formação Do Instituto Politécnico de Bragança," 151.

LITTO, Fredric M. 2014. "As Interfaces Da EAD Na Educação Brasileira." Revista USP 0 (100): 57. doi:10.11606/issn.2316-9036.v0i100p57-66.

MATTA, Cláudia Eliane da;Figueiredo, Ana Paula Silva. 2013. "Mooc : Transformação Das Práticas De," no. June 2013: 11-13. doi:10.13140/RG.2.1.1392.8086. 
MATTAR, João. 2011. "WEB 2.0 E REDES SOCIAIS NA EDUCAÇÃO A DIST NCIA:

CASES NO BRASIL." São Paulo: Cengage Learning, 1-23. http://www.educoas.org/portal/laeducacion2010.

MCAULEY, a, B Stewart, G Siemens, D Cormier, and Creative Commons. 2010. "The MOOC Model for Digital Practice." Massive Open Online Courses: Digital Ways of Knowing and Learning, 1-64. doi:10.1016/j.im.2011.09.007.

MENDES, Leonardo Manoel, RENATA KELLY DA SILVA, and CRISTINA LÚCIA FEIJÓ ORTOLANI. 2017. "MOOC - MASSIVE OPEN ONLINE COURSE COMO METODOLOGIA PARA EDUCAÇÃO EM SAÚDE: UMA AVALIAÇÃO BASEADA NAS EXPERIÊNCIAS DE ALUNOS QUE REALIZARAM CURSO APERFEIÇOAMENTO EM SAÚDE." In Apresentações Trabalhos Científicos. Associação Brasileira de Educação a Distância ABED. doi:10.17143/ciaed/XXIIICIAED.2017.00167.

PAPPANO, Laura. 2012. "The Year of the MOOC." The New York Times, 1-7. doi:10.1038/nmeth1154.

Pereira, Angela Maria de Almeida. 2015. "Uso de Recursos Educacionais Abertos (REA) $\mathrm{Na}$ Educação Superior/UAB: Sonho Ou Realidade?," 163. doi:10.1007/s13398-014-0173-7.2.

Silva, João Augusto Ramos e, Ronaldo Junior, and Fátima Oliveira. 2014. "MOOC: A Necessidade de Um Modelo de Negócio." Esud 2014, 243-55.

Souza, Costa De, Márcio Vieira de; Simon, Rangel Machado. 2014. "REDES SOCIAIS E MOOCs : ANÁLISE DE MÍDIAS PARA.” XI Congresso Brasileiro de Ensino Superior a DistâNcia, 5-8. 